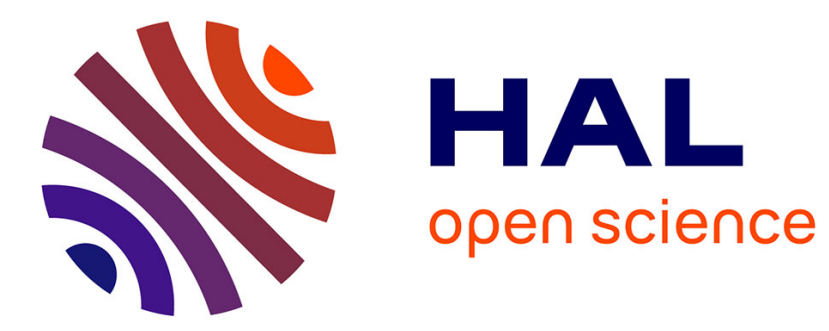

\title{
Exciting multi-DOF systems by feedback resonance
}

Denis Efimov, Alexander Fradkov, Tetsuya Iwasaki

\section{To cite this version:}

Denis Efimov, Alexander Fradkov, Tetsuya Iwasaki. Exciting multi-DOF systems by feedback resonance. Automatica, 2013, 49 (6), pp.1782-1789. 10.1016/j.automatica.2013.02.035 . hal-00813506

\section{HAL Id: hal-00813506 https://inria.hal.science/hal-00813506}

Submitted on 15 Apr 2013

HAL is a multi-disciplinary open access archive for the deposit and dissemination of scientific research documents, whether they are published or not. The documents may come from teaching and research institutions in France or abroad, or from public or private research centers.
L'archive ouverte pluridisciplinaire HAL, est destinée au dépôt et à la diffusion de documents scientifiques de niveau recherche, publiés ou non, émanant des établissements d'enseignement et de recherche français ou étrangers, des laboratoires publics ou privés. 


\title{
Exciting multi-DOF systems by feedback resonance
}

\author{
Denis Efimov ${ }^{a}$, Alexander Fradkov $^{\mathrm{b}}$, Tetsuya Iwasaki ${ }^{\mathrm{c}}$ \\ ${ }^{a}$ Non-A project at INRIA - LNE, Parc Scientifique de la Haute Borne \\ 40, avenue Halley Bât.A, Park Plaza 59650 Villeneuve d'Ascq, France \\ ${ }^{\mathrm{b}}$ Institute for Problems of Mechanical Engineering, 61, Bolshoy av., V.O. , St-Petersburg, 199178 Russia, \\ St.Petersburg State University and National Research University of Information Technology, Mechanics and Optics \\ ${ }^{\mathrm{c}}$ Department of Mechanical and Aerospace Engineering, University of California, Los Angeles, CA 99095
}

\begin{abstract}
The mechanism of entrainment to natural oscillations in a class of (bio)mechanical systems described by linear models is investigated. Two new nonlinear control strategies are proposed to achieve global convergence to a prescribed resonance mode of oscillation within a finite time. Effectiveness of the proposed methods for resonance entrainment is demonstrated by examples of computer simulation for linear and nonlinear systems.
\end{abstract}

Key words: Entrainment; nonlinear control; oscillations.

\section{Introduction}

The robotic or animal locomotion is a periodical movement synchronized with the environment influence. To minimize the energy consumption during the movement, the body oscillations have to be performed in a resonance mode. For example, the period of walking can be related to the natural frequency of a leg as a pendulum [Holt et al., 1991]. This fact is of great importance for analysis of the locomotion phenomenon in animals and for robotic system design as well. In this way a fundamental question arises: how to design a feedback controller ensuring reliable oscillations in (or near) a resonance mode?

An answer to this question is based on the fact that rhythmic animal motions are controlled by neural circuits called the Central Pattern Generator (CPG) [Delcomyn, 1980,Grillner et al., 1988], [Orlovsky et al., 1999]. CPGs are composed by neural networks generating sustained oscillations and defining the locomotion rhythm. Such a control mechanism of oscillations can be related to the entrainment phenomenon, when one oscillator (CPG) en-

\footnotetext{
^ This paper was not presented at any IFAC meeting

Email addresses: Denis.Efimov@inria.fr

(Denis Efimov), fradkov@mail.ru (Alexander Fradkov),

tiwasaki@ucla.edu (Tetsuya Iwasaki).
}

trains to the frequency and phase of rhythmic movement in another oscillator (in a series or feedback connection). This feedback mechanism is now used in robotics and mechanical systems to regulate locomotion [Futakata and Iwasaki, 2011], [Futakata and Iwasaki, 2008]. The conditions of resonance regime appearance are analyzed using (multivariable) Harmonic Balance (HB), and hence are approximate in nature.

The goal of this work is to make the next step and develop control methods that achieve resonance entrainment exactly and in a finite time (the system has to oscillate in a resonance mode after a finite time for any initial conditions). Our underlying idea is that not only the resonance motion is energyoptimal, but its control mechanism may also be a result of a natural optimization. We use the speed gradient principle [Fradkov, 2007] to arrange the type of behavior that was called feedback resonance in [Fradkov, 1999], [Andrievsky and Fradkov, 1999]. It is shown that the neural network implementation of the proposed algorithm can have a structure similar to that of [Futakata and Iwasaki, 2011], [Futakata and Iwasaki, 2008], though its functioning can be completely different.

The main results present two dynamical output controls for a class of multiple degree-of-freedom (DOF) mechanical systems: finite-time control (with an energy optimal excitation mechanism) and energy/phase regulator. The 
former achieves a prescribed mode of natural oscillations in a finite time, while the latter allows for regulation of the energy and phase for every mode. The properties of these control laws are precisely characterized with rigorous proofs. It is assumed that only position of the mechanical system is available for measurements.

Preliminary results are obtained in Section 2 where the resonance controls based on the speed gradient approach [Efimov and Fradkov, 2007b,Efimov and Fradkov, 2012], [Fradkov, 1999,Andrievsky and Fradkov, 1999] are introduced and applied to a single DOF linear pendulum. Optimality of a form of the speed gradient algorithm for energy excitation in the linear pendulum has been shown in [Andrievsky, 2005]. The problem statement of natural oscillation entrainment in linear mechanical multi-DOF systems is presented in Section 3, the relation between the entrainment of single DOF system and multi-DOF one is shown. The two control algorithms based on the results from [Efimov and Fradkov, 2007b,Efimov and Fradkov, 2012] are developed. Efficiency of the obtained control algorithms is demonstrated by examples of numerical simulation in Section 4. By computer simulation it is shown that the proposed control strategy can also be applied to resonance control of nonlinear systems.

\section{Preliminary results}

In this section we will develop basic control strategies by applying the results from [Andrievsky, 2005], [Efimov and Fradkov, 2007b,Efimov and Fradkov, 2012], [Fradkov, 1999,Andrievsky and Fradkov, 1999] to the linear model of a pendulum:

$$
\ddot{x}+\kappa \dot{x}+\omega^{2} x=b u,
$$

where $x \in \mathbb{R}$ is the angle of pendulum rotation, $\dot{x} \in \mathbb{R}$ is the angular velocity, $u \in[-U, U]$ is the control, $U \in \mathbb{R}_{+}$ is the maximal control amplitude; $\kappa \in \mathbb{R}_{+}$is the friction coefficient, $\omega \in \mathbb{R}_{+}$is the frequency of oscillations without friction, $b \in \mathbb{R}, b \neq 0$ is the control gain. It is assumed that $\omega>0.5 \kappa$, in this case the transfer function of the system (1) has poles $\lambda_{1,2}=-0.5 \kappa \pm \omega_{0} i$ with nonzero imaginary part $\omega_{0}=\sqrt{\omega^{2}-\kappa^{2} / 4}$, where $\omega_{0}$ is the natural frequency of oscillations for (1). Typically, the oscillations with the frequency $\omega_{0}$ are called resonant for (1). It is worth noting that the Bode magnitude plot of the system (1) reaches its maximum at the frequency $\omega_{1}=\sqrt{\omega^{2}-\kappa^{2} / 2}$ while $\omega \geq \kappa / \sqrt{2}$, and at the zero frequency otherwise (for the variable $\dot{x}$ the resonant frequency is $\omega)$. The difference between the frequencies $\omega, \omega_{0}, \omega_{1}$ is minor if the value of $\kappa$ is sufficiently small, as it is usually in practice and that is the case under consideration, i.e $\omega \gg \kappa / \sqrt{2}$.

Due to constraint on the control amplitude, the problem of excitation of the resonance oscillations becomes meaningful from practical point of view. If it is required to ensure oscillations with the maximal amplitude for a given amplitude of the control, then the resonance mode is a solution. The question is how to design such a control $u=u(x, \dot{x}),|u| \leq U$ providing the system oscillations with the frequency $\omega_{0}$ or $\omega_{1}$. An additional goal consists in the oscillation phase resetting.

\subsection{Controls for stabilization/excitation of the linear pendulum}

Let us first recall a simple control law that achieves convergence to the resonant oscillation in finite time. In the paper [Andrievsky, 2005] it is shown that the control

$$
u=U \operatorname{sign}(\dot{x})
$$

is the optimal solution of the terminal energy maximization problem. The solutions of the system (1) with the control (2) are understood in the Filippov sense [Filippov, 1988] (see also [Yakubovich et al., 2004] for a more general definition), roughly speaking it is an absolutely continuous function of time passed through the given initial conditions and satisfying the differential inclusion (1), (2) with

$$
\operatorname{sign}(s)= \begin{cases}s /|s| & \text { if } s \neq 0 \\ {[-1,1]} & \text { if } s=0\end{cases}
$$

the value $\operatorname{sign}(0)=0$ is used for simulations/experiments. Since the control (2) is piecewise constant, then solutions of the system (1), (2) can be presented as follows:

$$
\begin{aligned}
x(t)= & e^{-\kappa t / 2}\left[\omega_{0}^{-1}\left(\dot{x}(0)+\kappa\left\{x(0)-s_{0}\right\} / 2\right) \sin \left(\omega_{0} t\right)+\right. \\
& \left.+\left\{x(0)-s_{0}\right\} \cos \left(\omega_{0} t\right)\right]+s_{0}, \\
\dot{x}(t)= & e^{-\kappa t / 2}\left[\dot{x}(0) \cos \left(\omega_{0} t\right)-\omega_{0}^{-1}\left(\omega^{2}\left\{x(0)-s_{0}\right\}+\right.\right. \\
& \left.+\kappa \dot{x}(0) / 2) \sin \left(\omega_{0} t\right)\right], \\
s_{0}= & b \omega^{-2} U \operatorname{sign}(\dot{x}(0))
\end{aligned}
$$

for $t \in\left[0, t_{1}\right)$, where

$$
t_{1}=\frac{1}{\omega_{0}} \operatorname{atan}\left(\frac{\omega_{0} \dot{x}(0)}{\omega^{2}\left\{x(0)-s_{0}\right\}+\kappa \dot{x}(0) / 2}\right)
$$

is the instant of time when $\dot{x}\left(t_{1}\right)=0$ (for the first time). Introduce $t_{i}, i \geq 1$ as the sequence of instants satisfying constraints $\dot{x}\left(t_{i}\right)=0$ and $\dot{x}(t) \neq 0$ for $t \neq t_{i}, i \geq 1$. For $t \in\left[t_{i}, t_{i+1}\right), i \geq 1$ we have

$$
\begin{aligned}
x(t)= & e^{-\kappa\left(t-t_{i}\right) / 2}\left\{x\left(t_{i}\right)-s_{i}\right\}\left(\omega_{0}^{-1} \kappa / 2 \sin \left[\omega_{0}\left(t-t_{i}\right)\right]+\right. \\
& \left.+\cos \left[\omega_{0}\left(t-t_{i}\right)\right]\right)+s_{i}, \\
\dot{x}(t)= & -e^{-\kappa\left(t-t_{i}\right) / 2}\left\{x\left(t_{i}\right)-s_{i}\right\} \omega_{0}^{-1} \omega \sin \left[\omega_{0}\left(t-t_{i}\right)\right], \\
s_{i}= & -s_{i-1} .
\end{aligned}
$$


From the expression above we have that $t_{i+1}-t_{i}=\pi / \omega_{0}$, $i \geq 1$. Therefore, the control (2) ensures that the trajectory of system (1) converges to oscillations with the frequency $\omega_{0}$ [Efimov and Fradkov, 2007b] (the resonance oscillations) in the finite time $t_{1}$. The analysis reviewed above had previously lead to the following result.

Lemma 1 [Andrievsky, 2005,Efimov and Fradkov, 2007b] For the system (1) with the control (2) the following statements are true:

(1) convergence in the finite time $t_{1}$ to the oscillations with the frequency $\omega_{0}$;

(2) the asymptotic amplitude of oscillations for the variable $x$ is $\gamma_{0}=-b U / \omega^{2}\left(1+e^{-\kappa \pi / \omega_{0}}+\right.$ $\left.2 e^{-\kappa \pi /\left(2 \omega_{0}\right)}\right) /\left(1-e^{-\kappa \pi / \omega_{0}}\right)$.

Note that the control (2) is independent of values $\omega$ and $\kappa$ of the system parameters.

In the paper [Efimov and Fradkov, 2007b] an adaptive algorithm for amplitude adjustment in the control (2) is proposed ensuring a required amplitude of oscillations on the limit cycle $\gamma_{0}$ for the case of unknown values of the pendulum (1) parameters. In the paper [Efimov and Fradkov, 2007a] the same problem (excitation to the resonance mode of the pendulum (1) for unknown values of parameters) is solved using the adaptive observer technique. The control (2) is related with the mechanism of positive rate feedback with saturation discussed in [Futakata and Iwasaki, 2008].

The control law (2) does not regulate the phase of oscillations. In addition, the resulting oscillations in the resonance mode have non-constant energy $E$ :

$$
E(x, \dot{x})=0.5\left[\dot{x}^{2}+\omega^{2} x^{2}\right], \quad \dot{E}=-\kappa \dot{x}^{2}+b \dot{x} u .
$$

For $\kappa=0$, each fixed value of the energy $E$ corresponds to an oscillation with the angle amplitude $\sqrt{2 E} / \omega$, the presence of friction leads to the energy dissipation. The control presented in [Efimov and Fradkov, 2012] makes the regulation of both, the energy $E$ and the phase $\varphi \in[-\pi, \pi)$, for 1D and 2D lattices of linear oscillators without friction. Below, we will generalize the previous control law to deal with damped systems. The idea is based on the "action-angle" transformation of coordinates $(E, \varphi)$ :

$$
E=0.5\left[\dot{x}^{2}+\omega^{2} x^{2}\right], \varphi=\operatorname{atan}\left(\frac{\dot{x}}{\omega x}\right),
$$

which has well defined inverse

$$
x=\omega^{-1} \sqrt{2 E} \cos (\varphi), \dot{x}=\sqrt{2 E} \sin (\varphi) .
$$

In the new coordinates the pendulum equations (1) can be rewritten as follows:

$$
\begin{aligned}
\dot{E} & =\sqrt{2 E} \sin (\varphi)[b u-\kappa \sqrt{2 E} \sin (\varphi)] \\
\dot{\varphi} & =\cos (\varphi) b u / \sqrt{2 E}-\kappa \cos (\varphi) \sin (\varphi)-\omega
\end{aligned}
$$

which are well defined for any $E>0$ (if $E=0$, then the system is at the origin). Let $E_{d}>0$ and $\varphi_{d}(t)=\phi_{d}-\omega t$, $\phi_{d} \in[-\pi, \pi)$ be the desired energy and phase values respectively, then the control $u$ can be chosen in the form:

$$
u= \begin{cases}b^{-1} \kappa \sqrt{2 E} \sin (\varphi)-\rho_{1}\left(\psi_{1}\right) & \text { if } V \leq 0.5 E_{d}^{2} \\ b^{-1} \kappa \sqrt{2 E} \sin (\varphi)-\rho_{2}\left(\psi_{2}\right) & \text { if }(V, E) \in \Omega_{V, E} \\ -\rho_{2}\left(\psi_{2}\right) & \text { otherwise }\end{cases}
$$

$$
\begin{aligned}
\Omega_{V, E} & =\left\{(V, E) \in \mathbb{R}_{+}^{2}: V>0.5 E_{d}^{2}, E \leq E_{d}\right\}, \\
V & =0.5\left[\left(E-E_{d}\right)^{2}+E_{d}^{2} \pi^{-2}\left(\varphi-\varphi_{d}(t)\right)^{2}\right], \\
\psi_{1} & =\psi_{2}+b E_{d}^{2} \pi^{-2}\left(\varphi-\varphi_{d}(t)\right) \cos (\varphi) / \sqrt{2 E}, \\
\psi_{2} & =b\left(E-E_{d}\right) \sqrt{2 E} \sin (\varphi),
\end{aligned}
$$

where $\psi_{i} \rho_{i}\left(\psi_{i}\right)>0, i=1,2$ for any $\psi_{i} \neq 0$ and $\left|\rho_{1}\left(\psi_{1}\right)\right| \leq U-2 b^{-1} \kappa \sqrt{E_{d}},\left|\rho_{2}\left(\psi_{2}\right)\right| \leq U-b^{-1} \kappa \sqrt{2 E_{d}}$ (it is assumed that $U>2 b^{-1} \kappa \sqrt{E_{d}}$ ).

Lemma 2 For the system (1), the control (3) ensures boundedness of solutions with the limit relations

$$
\lim _{t \rightarrow+\infty} E(t)=E_{d}, \quad \lim _{t \rightarrow+\infty}\left[\varphi(t)-\varphi_{d}(t)\right]=0
$$

for any $E(0) \in \mathbb{R}_{+}$and $\varphi(0) \in[-\pi, \pi)$, in addition $|u| \leq$ $U$.

PROOF. First, assume that $V\left(t^{\prime}\right) \leq 0.5 E_{d}^{2}$ for some $t^{\prime} \geq 0$ and consider the Lyapunov function $V$ behavior for the system (1) and the control (3) for $t \geq t^{\prime}$, we obtain:

$$
\dot{V}=-\psi_{1} \rho_{1}\left(\psi_{1}\right) \leq 0 .
$$

Therefore, $V(t) \leq V\left(t^{\prime}\right) \leq 0.5 E_{d}^{2}$ for all $t \geq t^{\prime}$. Since $\left|\varphi-\varphi_{d}(t)\right| \leq \pi$ by the phase definition, then the relation $V(t) \leq 0.5 E_{d}^{2}$ implies that $E(t) \leq 2 E_{d}$ for all $t \geq t^{\prime}$. From the control (3) definition, for $V \leq 0.5 E_{d}^{2}$ we get that $|u| \leq U$ in this case. Next, the errors $E-E_{d}$ and $\varphi-$ $\varphi_{d}(t)$ stay bounded for all $t \geq 0$. This fact, for any finite value $E_{d}$, implies boundedness of the state variables $x(t)$ and $\dot{x}(t)$. According to the function $\rho_{1}$ properties, $\dot{V}=$ 0 if and only if $\psi_{1}=0$. Since all trajectories of the system are bounded, asymptotically they converge to an invariant set where $\psi_{1}=0$. The desired asymptotic convergence $E(t) \rightarrow E_{d}$ and $\varphi(t) \rightarrow \varphi_{d}(t)$ follows the observability property: $\psi_{1}(t)=0$ for $\forall t \geq 0$ implies that $V(t)=0$. Indeed, assume that $\psi_{1}(t)=0, V(t) \leq 0.5 E_{d}^{2}$ for $\forall t \geq 0$, then $u=b^{-1} \kappa \sqrt{2 E} \sin (\varphi)$ and the system 
(1) with this control can be simplified as follows:

$\dot{E}=0, \dot{\varphi}=-\omega$.

Therefore, $E(t)=E_{0}$ and $\varphi(t)=\phi_{0}-\omega t$ for all $t \geq 0$ for some $E_{0} \geq 0$ and $\phi_{0} \in[-\pi, \pi)$, then

$$
\begin{aligned}
\psi_{1}(t)= & b\left[\left(E_{0}-E_{d}\right) \sqrt{2 E_{0}} \sin \left(\phi_{0}-\omega t\right)\right. \\
& \left.+E_{d}^{2} \pi^{-2}\left(\phi_{0}-\phi_{d}\right) \cos \left(\phi_{0}-\omega t\right) / \sqrt{2 E_{0}}\right]=0
\end{aligned}
$$

for all $t \geq 0$ by the assumption. However, the last quantity can be true only for $E_{0}=E_{d}$ and $\phi_{0}=\phi_{d}$, that is necessary to prove (the relations (4) are satisfied).

Second, consider the general case when $V(t)>0.5 E_{d}^{2}$ for $t \geq 0$. Consider an auxiliary Lyapunov function $W=0.5\left(E-E_{d}\right)^{2}$, which derivative has the form:

$$
\begin{aligned}
\dot{W}= & \left(E-E_{d}\right) \sqrt{2 E} \sin (\varphi)\left\{b\left[v-\rho_{2}\left(\psi_{2}\right)\right]\right. \\
& -\kappa \sqrt{2 E} \sin (\varphi)\}
\end{aligned}
$$

where $v=0$ for $E>E_{d}$ and $v=b^{-1} \kappa \sqrt{2 E} \sin (\varphi)$ for $E \leq E_{d}$. If $E \leq E_{d}$, then $\dot{W}=-\psi_{2} \rho_{2}\left(\psi_{2}\right)$; if $E>E_{d}$, then

$$
\dot{W}=-\psi_{2} \rho_{2}\left(\psi_{2}\right)-2 \kappa\left(E-E_{d}\right) E \sin ^{2}(\varphi) \leq-\psi_{2} \rho_{2}\left(\psi_{2}\right)
$$

therefore, $\dot{W}=-\psi_{2} \rho_{2}\left(\psi_{2}\right) \leq 0$ whenever $V>0.5 E_{d}^{2}$. Due to the form of $W$ and $\psi_{2}$, the energy $E(t)$ stay bounded, the error $\left|E(t)-E_{d}\right|$ is not increasing and $|u| \leq U$ in this case also. Using partial detectability arguments [Shiriaev, 2000,Shiriaev and Fradkov, 2001] we can prove that in this case $\lim _{t \rightarrow+\infty} E(t)=E_{d}$ (if $V(t)>$ $0.5 E_{d}^{2}$ for all $\left.t \geq 0\right)$. However, if $E\left(t^{\prime}\right)=E_{d}$, then $V\left(t^{\prime}\right) \leq$ $0.5 E_{d}^{2}$. Consequently, either $\lim _{t \rightarrow+\infty}\left[\varphi(t)-\varphi_{d}(t)\right]$ under the condition $V(t)>0.5 E_{d}^{2}$, which is satisfied for all $t \geq 0$ (and the stabilization goals (4) are achieved simultaneously), or $\varphi(t) \neq \varphi_{d}(t)$ and there is a finite time instant $t^{\prime} \geq 0$ such that $V\left(t^{\prime}\right) \leq 0.5 E_{d}^{2}$. Next, according to the consideration above $V(t) \leq 0.5 E_{d}^{2}$ for all $t \geq t^{\prime}$ and the required limit relations $(\overline{4})$ hold. In both cases the variables $x$ and $\dot{x}$ stay bounded under $|u| \leq U$.

It is worth to stress that if $E\left(t^{\prime}\right)=0$ for some $t^{\prime} \geq 0$, then it becomes possible to have $\left|\psi_{1}\left(t^{\prime}\right)\right|=\infty$, but due to the stated boundedness of $\rho_{1}$, the control input stays finite. In this case the control (3) generates an exciting pulse, which pushes the trajectory away from the origin $\left(E\left(t^{\prime}\right)=0\right)$.

Later in this paper, the controls (2), (3) will be used to excite a resonance mode of oscillations in a finite time for multi-DOF systems. When achieving the resonance entrainment to a prescribed mode, it is important to attenuate the other oscillation modes at the same time. For this purpose, we are also interested in the finite-time stabilization of the system (1). In applications, an asymptotic achievement of a control goal may not be considered satisfactory when one has to pass from one task to another within a finite-time frame. During robotic locomotion for instance, it may be required that switching from walking to running be completed after a finite-time transient. At a conceptual level, such requirement may be formalized as a finite-time transition from one mode of resonance oscillation to another. To this end, we propose the following control to achieve finite-time convergence of every trajectory of (1) to the origin:

$$
u=-\beta \operatorname{sign}(\dot{x})-(\beta+\varepsilon) \operatorname{sign}\left(\kappa \dot{x} / \omega^{2}+x\right),
$$

where $2 \beta+\varepsilon=U$ and $\beta>0, \varepsilon>0$ are the design parameters.

Lemma 3 The control (5) ensures global stabilization of the origin for the system (1) in a finite time $t_{0} \geq 0$ :

$$
t_{0} \leq T_{0}=2 \mu^{-1} \sqrt{V\left[\kappa \dot{x}(0) / \omega^{2}+x(0), \dot{x}(0)\right]},
$$

where

$$
\begin{aligned}
V\left(\xi_{1}, \xi_{2}\right)= & 0.5\left[0.5 \omega^{2} \xi_{1}^{2}+\xi_{2}^{2}+0.5 \omega^{2}\left(\xi_{1}-\kappa \xi_{2} / \omega^{2}\right)^{2}\right] \\
& +b(\beta+\varepsilon)\left|\xi_{1}\right|
\end{aligned}
$$

and

$$
\begin{aligned}
\mu= & \min \{b(\beta+\varepsilon) \kappa / \omega, b \beta \omega /(\sqrt{0.5} \omega+0.5 \kappa) \\
& \left.0.5 \kappa \omega^{2} / \sqrt{b(\beta+\varepsilon)}, 8 \kappa b \sqrt{b(\beta+\varepsilon)} \varepsilon /\left[3 \sqrt[3]{2} \omega^{2}\right]\right\}
\end{aligned}
$$

PROOF. Consider the following coordinate transformation for the system (1) and the control (5):

$$
\begin{aligned}
\xi_{1} & =\kappa \dot{x} / \omega^{2}+x, \quad \xi_{2}=\dot{x} ; \\
\dot{\xi}_{1} & =\kappa\left[-\omega^{2} \xi_{1}+b u\right] / \omega^{2}+\xi_{2}, \\
\dot{\xi}_{2} & =-\omega^{2} \xi_{1}+b u ; \\
u & =-\beta \operatorname{sign}\left(\xi_{2}\right)-(\beta+\varepsilon) \operatorname{sign}\left(\xi_{1}\right) .
\end{aligned}
$$

Consider the Lyapunov function $V\left(\xi_{1}, \xi_{2}\right)$ for this system, then

$$
\begin{aligned}
\dot{V}= & 0.5 \omega^{2} \xi_{1}\left(\kappa\left[-\omega^{2} \xi_{1}+b u\right] / \omega^{2}+\xi_{2}\right)+ \\
& \xi_{2}\left(-\omega^{2} \xi_{1}+b u\right)+ \\
& 0.5 \omega^{2}\left(\xi_{1}-\kappa \xi_{2} / \omega^{2}\right)\left(\kappa \left[-\omega^{2} \xi_{1}+\right.\right. \\
& \left.b u] / \omega^{2}+\xi_{2}-\kappa\left[-\omega^{2} \xi_{1}+b u\right] / \omega^{2}\right)+ \\
& b(\beta+\varepsilon) \operatorname{sign}\left(\xi_{1}\right)\left(\kappa\left[-\omega^{2} \xi_{1}+b u\right] / \omega^{2}+\xi_{2}\right) \\
= & -0.5 \kappa \omega^{2} \xi_{1}^{2}-0.5 \kappa \xi_{2}^{2}-b(\beta+\varepsilon) \kappa\left|\xi_{1}\right|+ \\
& 0.5 \kappa b \xi_{1} u+b \xi_{2} u+ \\
& \kappa b^{2}(\beta+\varepsilon) \operatorname{sign}\left(\xi_{1}\right) u / \omega^{2}+b(\beta+\varepsilon) \operatorname{sign}\left(\xi_{1}\right) \xi_{2}
\end{aligned}
$$




$$
\begin{aligned}
= & -0.5 \kappa \omega^{2} \xi_{1}^{2}-0.5 \kappa \xi_{2}^{2}-1.5 b(\beta+\varepsilon) \kappa\left|\xi_{1}\right|- \\
& 0.5 \beta \kappa b \xi_{1} \operatorname{sign}\left(\xi_{2}\right)-b \beta\left|\xi_{2}\right|+ \\
& \kappa b^{2}(\beta+\varepsilon)\left[-\beta \operatorname{sign}\left(\xi_{1}\right) \operatorname{sign}\left(\xi_{2}\right)-\right. \\
& \left.(\beta+\varepsilon) \operatorname{sign}\left(\xi_{1}\right)^{2}\right] / \omega^{2} \\
\leq & -0.5 \kappa \omega^{2} \xi_{1}^{2}-0.5 \kappa \xi_{2}^{2}-1.5 b(\beta+\varepsilon) \kappa\left|\xi_{1}\right|+ \\
& 0.5 \beta \kappa b\left|\xi_{1}\right|-b \beta\left|\xi_{2}\right|+\kappa b^{2}(\beta+\varepsilon)\left[\beta \operatorname{sign}\left(\xi_{1}\right)^{2}-\right. \\
& \left.(\beta+\varepsilon) \operatorname{sign}\left(\xi_{1}\right)^{2}\right] / \omega^{2} \\
\leq & -0.5 \kappa \omega^{2} \xi_{1}^{2}-0.5 \kappa \xi_{2}^{2}-b(\beta+\varepsilon) \kappa\left|\xi_{1}\right|- \\
& b \beta\left|\xi_{2}\right|-\kappa b^{2}(\beta+\varepsilon) \varepsilon \operatorname{sign}\left(\xi_{1}\right)^{2} / \omega^{2} .
\end{aligned}
$$

This inequality implies global asymptotic stability of the system. To prove the finite time stability note that $\sqrt{a+b} \leq \sqrt{a}+\sqrt{b}$ for any $a, b \in \mathbb{R}_{+}$, then

$$
\begin{aligned}
\sqrt{V} \leq & 0.5 \omega\left|\xi_{1}\right|+\sqrt{0.5}\left|\xi_{2}\right|+ \\
& 0.5 \omega\left|\xi_{1}-\kappa \xi_{2} / \omega^{2}\right|+\sqrt{b(\beta+\varepsilon)} \sqrt{\left|\xi_{1}\right|} \\
\leq & \omega\left|\xi_{1}\right|+(\sqrt{0.5}+0.5 \kappa / \omega)\left|\xi_{2}\right|+\sqrt{b(\beta+\varepsilon)} \sqrt{\left|\xi_{1}\right|}
\end{aligned}
$$

Using the relation $\sqrt{|a|} \leq 3 \sqrt[3]{2} / 8 \operatorname{sign}(a)^{2}+a^{2}$ that is valid for any $a \in \mathbb{R}$ we finally obtain

$$
\begin{aligned}
\sqrt{V} \leq \omega\left|\xi_{1}\right|+(\sqrt{0.5}+0.5 \kappa / \omega)\left|\xi_{2}\right|+ \\
\sqrt{b(\beta+\varepsilon)}\left[3 \sqrt[3]{2} / 8 \operatorname{sign}\left(\xi_{1}\right)^{2}+\xi_{1}^{2}\right]
\end{aligned}
$$

Then for the defined $\mu$ we have

$$
\dot{V} \leq-\mu \sqrt{V}
$$

The corresponding solution in the time domain satisfies the inequality for all $t \geq 0$ :

$$
V(t) \leq(\sqrt{V(0)}-0.5 \mu t)^{2}
$$

Since $V(t) \geq 0$, the function $V(t)$ has to reach the zero in a time $t_{0}$ smaller than the time $T_{0}$.

\subsection{Differentiation}

Frequently in applications only the angle position $x$ is available for measurements while the controls (2), (5) and (3) are based on the velocity $\dot{x}$. We will use the following nonlinear filter, which has been proposed in [Efimov and Fridman, 2011] to evaluate $\dot{x}$ in a finite time.

Lemma 4 [Efimov and Fridman, 2011] Let $|\dot{x}(t)| \leq$ $L_{1} \in \mathbb{R}_{+},|\ddot{x}(t)| \leq L_{2} \in \mathbb{R}_{+}$, for all $t \geq 0$. Then the filter

$$
\begin{gathered}
\dot{\zeta}_{1}=-\alpha \sqrt{\left|\zeta_{1}-x(t)\right|} \operatorname{sign}\left[\zeta_{1}-x(t)\right]+\zeta_{2}, \\
\dot{\zeta}_{2}=-\varpi \operatorname{sign}\left[\zeta_{1}-x(t)\right]-\chi \operatorname{sign}\left(\zeta_{2}\right)-\zeta_{2}, \\
\zeta_{1}(0)=x(0), \quad \zeta_{2}(0)=0,
\end{gathered}
$$

where

$$
\begin{gathered}
\chi=0.25 \sqrt[4]{2} L_{1}+\varrho, \varpi>L_{1}+L_{2}+3 \chi, \\
\alpha=4\left[\sqrt{2\left(\varpi+L_{1}+L_{2}+2 \chi\right)} \chi+\right. \\
\left.\sqrt{\varpi+L_{1}+L_{2}+3 \chi}\left(L_{1}+L_{2}+2 \chi\right)\right] / \\
{\left[2 \varpi-L_{1}-L_{2}-2 \chi\right]}
\end{gathered}
$$

for some $\varrho \in \mathbb{R}_{+}$, has bounded solutions and $\zeta_{2}(t)=\dot{x}(t)$ for all $t \geq T_{d}$ with $T_{d} \leq L_{1} /\left(0.25 \sqrt[4]{2} L_{1}+\varrho\right)$.

\section{Main results}

This section extends the feedback resonance mechanism of the controls (2) and (3) for the single-DOF system to the multi-DOF system:

$$
J \ddot{x}+D \dot{x}+S x=B u, y=C x,
$$

where $x \in \mathbb{R}^{n}$ is the vector of generalized coordinates, $u \in \mathbb{R}^{m}$ and $y \in \mathbb{R}^{m}$ are the input and the output respectively.

Assumption 5 The inertia, damping and stiffness matrices satisfy

$$
\begin{aligned}
J= & J^{T}>0, S=S^{T} \geq 0, D=\varrho S, \varrho>0, \\
& \operatorname{rank}(B)=\operatorname{rank}(C)=m, m=n .
\end{aligned}
$$

The equation (8) with Assumption 5 captures a fairly general class of standard mechanical systems with mass, stiffness, and damping elements, linearized around an equilibrium point. Two restrictions are imposed by Assumption 5 . One is that $B$ and $C$ are square invertible, implying that all the degrees of freedom are fully actuated and measured. The other is the Rayleigh damping $D=\varrho S$, which is commonly used in structural dynamics to capture (uncertain) dissipation effects. These assumptions make the analysis simple enough to provide insights into the natural entrainment mechanism.

In addition, the conditions in Assumption 5 are frequently met in biological systems [Futakata and Iwasaki, 2011], [Futakata and Iwasaki, 2008]. A musculo-skeletal body can be viewed as a set of rigid links connected by rotational joints. At each joint, the muscle and tendon serve as an origin of visco-elastic property being also a torque actuator. The muscle length is fed back to the motor control system through stretch receptors, and the collocation arises naturally in biomechanics. In this case, the stiffness $S$ and the damping $D$ matrices share the same structure.

A natural mode of (8) is defined by a pair of the natural frequency $\omega_{i} \in \mathbb{R}_{+}$and the mode shape $\xi_{i} \in \mathbb{R}^{n}$ 
satisfying $\left(S-\omega_{i}^{2} J\right) \xi_{i}=0, i=\overline{1, n}$ (where the symbol $\overline{1, n}$ is used to denote the sequence of integers $1,2, \ldots, n)$. For brevity of presentation we will assume that all $\omega_{i}$, $i=\overline{1, n}$ are different (the approach can be easily extended to the multiple case). We will also assume that the controller is allowed to have centralized information processing.

\subsection{Natural oscillation entrainment problem}

The problem of natural oscillation entrainment can be formulated now as follows. Design a control $u$ ensuring that the system (8) oscillates at a selected natural frequency $\omega_{k}, k \in\{1, \ldots, n\}$.

Our approach is based on the transformation $x=P z$ of the system (8) to the normal mode canonical form:

$$
\ddot{z}+K \dot{z}+\Omega z=R u
$$

where

$$
\begin{aligned}
& \Omega=P^{-1} J^{-1} S P=\operatorname{diag}\left[\omega_{1}^{2}, \ldots, \omega_{n}^{2}\right], \\
& K=P^{-1} J^{-1} D P=\operatorname{diag}\left[\kappa_{1}, \ldots, \kappa_{n}\right], \\
& R=P^{-1} J^{-1} B, \quad \kappa_{i}=\varrho \omega_{i}^{2}, \quad i=\overline{1, n}
\end{aligned}
$$

and the columns of the matrix $P$ are composed of the vectors $\xi_{i}$. Under Assumption 5 we may assume that the vector $x=C^{-1} y$ is available for measurements (the matrix $C$ has rank $n$ ). By the same arguments, since $\operatorname{rank}(B)=n$, the matrix $R$ is nonsingular and introducing an auxiliary control $v=R u$ we may rewrite the system (9) as a sequence of independent systems (1):

$$
\ddot{z}_{i}+\kappa_{i} \dot{z}_{i}+\omega_{i}^{2} z_{i}=v_{i}, i=\overline{1, n} .
$$

Thus to choose $v_{i}, i=\overline{1, n}$ the controls (2), (5) and (3) can be used. The $i$-th system in (10) is responsible for the system (8) oscillation on the frequency $\omega_{i}$ or $\omega_{i, 0}=\sqrt{\omega_{i}^{2}-\kappa_{i}^{2} / 4}$. Stabilization of the $i$-th normal mode at the origin (zero energy level) implies elimination of the frequency $\omega_{i}$ from the frequency spectrum of the system (8) oscillation. Thus to solve the problem of natural oscillation entrainment to the frequency $\omega_{k}$, it is necessary to stabilize at the origin all normal modes with $\omega_{i} \neq \omega_{k}$ providing a sufficient level of excitation for the $k$-th mode. For future references define the set of canceling normal modes $\mathcal{J}=\{1, \ldots, n\} \backslash\{k\}$.

\subsection{The finite-time control}

We choose the control (2) to excite the $k$-th mode, and the control (5) to attenuate the other modes. The differentiator (6) is used to calculate velocities.
Theorem 6 Let Assumption 5 be satisfied and a constant $U>0$ be given. Then the control

$$
\begin{gathered}
u=R^{-1} v, v=\left[v_{1}, \ldots, v_{n}\right]^{T}, \\
v_{k}=U \operatorname{sign}\left(\hat{\dot{z}}_{k}\right), \\
v_{j}=-\beta \operatorname{sign}\left(\hat{\dot{z}}_{j}\right)- \\
(\beta+\varepsilon) \operatorname{sign}\left(\kappa_{j} \hat{\dot{z}}_{j} / \omega_{j}^{2}+z_{j}\right), j \in \mathcal{J} ;
\end{gathered}
$$

where $2 \beta+\varepsilon=U$ and

$$
\begin{aligned}
\hat{\dot{z}}_{i}= & \zeta_{2, i} ; \\
\dot{\zeta}_{1, i}= & -\alpha_{i} \sqrt{\left|\zeta_{1, i}-z_{i}(t)\right|} \operatorname{sign}\left[\zeta_{1, i}-z_{i}(t)\right]+\zeta_{2, i}, \\
\dot{\zeta}_{2, i}= & -\varpi_{i} \operatorname{sign}\left[\zeta_{1, i}-z_{i}(t)\right]-\chi_{i} \operatorname{sign}\left(\zeta_{2, i}\right)-\zeta_{2, i}, \\
& \zeta_{1, i}(0)=z_{i}(0), \zeta_{2, i}(0)=0, \quad i=\overline{1, n}
\end{aligned}
$$

with the parameters $\alpha_{i}, \varpi_{i}$ and $\chi_{i}$ calculated in accordance with (7) for

$$
\begin{gathered}
L_{1, i}=\sqrt{2 \epsilon_{i} /\left(\eta_{i} \nu_{i}\right)} U, L_{2, i}=\left(\kappa_{i}+\omega_{i}^{2}\right) L_{1, i}+U, \\
\eta_{i}=\min \left\{0.5,0.25 \omega_{i}^{2}\right\}, \\
\nu_{i}=\kappa_{i} \min \left\{\frac{1}{3}, \frac{0.5}{1+\omega_{i}^{-2} \kappa_{i}^{2}}\right\}, \epsilon_{i}=0.25 \kappa_{i} \omega_{i}^{-2}+\kappa_{i}^{-1},
\end{gathered}
$$

ensures for the system (8) boundedness of solutions and the natural oscillation entrainment for all initial conditions in a finite time.

PROOF. The control $u$ and the auxiliary inputs $v_{i}$, $i=\overline{1, n}$ are always bounded by construction. Then the solutions of the systems (10) are bounded for all $i=\overline{1, n}$ (since $\kappa_{i}>0$ and $\omega_{i}^{2}>0$ ). Moreover, for any initial conditions the trajectories of (10) have to converge in a finite time to the set where $\left|\dot{z}_{i}\right| \leq L_{1, i}$ and $\left|\ddot{z}_{i}\right| \leq L_{2, i}$, $i=\overline{1, n}$. Indeed, for the Lyapunov function $S\left(z_{i}, \dot{z}_{i}\right)=$ $0.5\left[0.5 \omega_{i}^{2}\left(z_{i}+\kappa_{i} \dot{z}_{i} / \omega_{i}^{2}\right)^{2}+\dot{z}_{i}^{2}+0.5 \omega_{i}^{2} z_{i}^{2}\right]$ we have

$$
\begin{aligned}
\dot{S} & \leq-0.25 \omega_{i}^{2} \kappa_{i}\left(z_{i}+\kappa_{i} \dot{z}_{i} / \omega_{i}^{2}\right)^{2}-0.25 \kappa_{i} \dot{z}_{i}^{2}+\epsilon_{i} v_{i}^{2} \\
& \leq-\nu_{i} S+\epsilon_{i} v^{2}
\end{aligned}
$$

which for $\mathbf{z}_{i}=\left[z_{i} \dot{z}_{i}\right]^{T}$ implies $\eta_{i}\left\|\mathbf{z}_{i}(t)\right\|^{2} \leq 0.5 \dot{z}_{i}^{2}(t)+$ $0.25 \omega_{i}^{2} z_{i}^{2}(t) \leq S(t) \leq 2 \nu_{i}^{-1} \epsilon_{i} v_{i}^{2}$ for all $t \geq t_{c}, t_{c} \in \mathbb{R}_{+}$. Therefore, $\left\|\mathbf{z}_{i}(t)\right\| \leq \sqrt{2 \epsilon_{i} /\left(\eta_{i} \nu_{i}\right)} U$ and

$$
\begin{aligned}
& \left|\dot{z}_{i}(t)\right| \leq \sqrt{2 \epsilon_{i} /\left(\eta_{i} \nu_{i}\right)} U=L_{1, i}, \\
& \left|\ddot{z}_{i}(t)\right| \leq\left(\kappa_{i}+\omega_{i}^{2}\right) L_{1, i}+U=L_{2, i}
\end{aligned}
$$

for all $t \geq t_{c}$. In this case according to Lemma 4 there is a finite time $T_{d} \geq t_{c}$ such that $\hat{\dot{z}}_{i}=\zeta_{2, i}, i=\overline{1, n}$, and the control $v_{k}=U \operatorname{sign}\left(\hat{\dot{z}}_{k}\right)$ is identical to (1), the controls $v_{j}=-\beta \operatorname{sign}\left(\hat{\dot{z}}_{j}\right)-(\beta+\varepsilon) \operatorname{sign}\left(\kappa_{j} \hat{\dot{z}}_{j} / \omega_{j}^{2}+z_{j}\right)$ for 


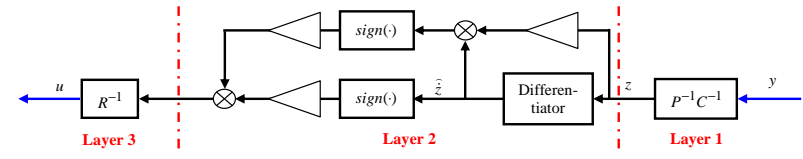

Fig. 1. The finite time control scheme

all $j \in \mathcal{J}$ coincide with (5). Thus from Lemmas 1 and 3 there are time instants $t_{1, k} \geq T_{d}$ and $t_{0, j} \geq T_{d}$ such that the $k$-th mode oscillates on the frequency $\omega_{k, 0}$ for $t \geq t_{1, k}$, while all other modes are canceled for $t \geq t_{0, j}$.

In this theorem the upper limit of the control $U$ is introduced for $v$ for brevity of formulation (it can be recalculated from an upper bound of the control $u$ ). Unlike the algorithms of [Futakata and Iwasaki, 2011], [Futakata and Iwasaki, 2008], the control methods proposed in this paper achieve resonance entrainment exactly and in a finite time with an optimal performance. These advantages are based on a special structure of the controls (2), (5) and a nonlinear differentiation algorithm used for estimation of the derivatives $\hat{\dot{z}}_{j}$.

This control has a neural network interpretation given in Fig. 1. As in [Futakata and Iwasaki, 2011] the network has linear input and output layers and the nonlinear CPG hidden layer.

\subsection{The energy/phase regulation}

Let $E_{d}>0$ be the desired value of energy $E_{k}=0.5\left[\dot{z}_{k}^{2}+\right.$ $\left.\omega_{k}^{2} z_{k}^{2}\right]$ for the $k$-th mode excitation and $\varphi_{d}(t)=\phi_{d}-\omega t$, $\phi_{d} \in[-\pi, \pi)$ be the desired trajectory of the phase for this mode. In this case the control (3) can be applied to the $k$-th mode for its excitation and phase resetting. For the $j$-th modes with $j \in \mathcal{J}$, to attenuate the oscillations, the control (5) can be used as before. The differentiator (6) calculates the estimates of velocities.

Theorem 7 Let Assumption 5 be satisfied and a constant $U>2 \kappa_{k} \sqrt{E_{d}}$ be given. Consider the control

$$
\begin{gathered}
u=R^{-1} v, v=\left[v_{1}, \ldots, v_{n}\right]^{T}, \\
v_{k}= \begin{cases}\kappa_{k} \hat{\dot{z}}_{k}-\rho_{1}\left(\hat{\psi}_{1}\right) & \text { if } \hat{V} \leq 0.5 E_{d}^{2}, \\
\hat{\dot{z}}_{k}-\rho_{2}\left(\hat{\psi}_{2}\right) & \text { if } \hat{V}>0.5 E_{d}^{2} \text { and } \hat{E}_{k} \leq E_{d}, \\
-\rho_{2}\left(\hat{\psi}_{2}\right) & \text { if } \hat{V}>0.5 E_{d}^{2} \text { and } \hat{E}_{k}>E_{d},\end{cases} \\
\hat{V}=0.5\left[\left(\hat{E}_{k}-E_{d}\right)^{2}+E_{d}^{2} \pi^{-2}\left(\hat{\varphi}_{k}-\varphi_{d}(t)\right)^{2}\right], \\
\hat{\psi}_{1}=\hat{\psi}_{2}+0.5 \omega_{k} E_{d}^{2} \pi^{-2}\left(\hat{\varphi}_{k}-\varphi_{d}(t)\right) z_{k} / \hat{E}_{k}, \\
\hat{\psi}_{2}=\left(\hat{E}_{k}-E_{d}\right) \hat{\dot{z}}_{k}, \\
\hat{E}_{j}=-\beta \operatorname{sign}\left(\hat{\dot{z}}_{j}\right)-(\beta+\varepsilon) \operatorname{sign}\left(\kappa_{j} \hat{\dot{z}}_{j} / \omega_{j}^{2}+\omega_{k}^{2} z_{k}^{2}\right], \hat{\varphi}_{k}=a t a n\left(\frac{\hat{\dot{z}}_{k}}{\omega_{k} z_{k}}\right),
\end{gathered}
$$

where the functions $\rho_{i}, i=1,2$ are chosen to satisfy $\hat{\psi}_{i} \rho_{i}\left(\hat{\psi}_{i}\right)>0$ for any $\hat{\psi}_{i} \neq 0,\left|\rho_{1}\left(\hat{\psi}_{1}\right)\right| \leq U-2 \kappa_{k} \sqrt{E_{d}}$, $\left|\rho_{2}\left(\hat{\psi}_{2}\right)\right| \leq U-\kappa_{k} \sqrt{2 E_{d}}$, and the estimates $\hat{\dot{z}}_{i}$ are generated by the differentiators (12) with the parameters $\alpha_{i}$, $\varpi_{i}$ and $\chi_{i}$ calculated in accordance with (7), (13). The control (14) ensures boundedness of the system (8) solutions and the natural oscillation entrainment with the phase regulation for all initial conditions.

PROOF. Again, the control $u$ and the auxiliary inputs $v_{i}, i=\overline{1, n}$ are always bounded by construction $\left(\left|v_{i}\right| \leq\right.$ $U)$. Then the solutions of the systems (10) are bounded for all $i=\overline{1, n}$ and for any initial conditions the trajectories of (10) have to converge in a finite time to the set where $\left|\dot{z}_{i}\right| \leq L_{1, i}$ and $\left|\ddot{z}_{i}\right| \leq L_{2, i}, i=\overline{1, n}$ (the constants $L_{1, i}, L_{2, i}$ are specified in (13), this can be proven using the Lyapunov function $S$ as before). In this case according to Lemma 4 there is a finite time $T_{d} \geq t_{c}$ such that $\hat{\dot{z}}_{i}=\zeta_{2, i}, i=\overline{1, n}$, and the control $v_{k}$ is identical to (3), the controls $v_{j}=-\beta \operatorname{sign}\left(\hat{\dot{z}}_{j}\right)-(\beta+\varepsilon) \operatorname{sign}\left(\kappa_{j} \hat{\dot{z}}_{j} / \omega_{j}^{2}+z_{j}\right)$ for all $j \in \mathcal{J}$ coincide with (5). Thus from Lemmas 2 and 3 there are time instants $t_{0, j} \geq T_{d}$ such that the modes in $\mathcal{J}$ are canceled for all $t \geq t_{0, j}$, in addition

$$
\lim _{t \rightarrow+\infty} E_{k}(t)=E_{d}, \quad \lim _{t \rightarrow+\infty}\left[\varphi_{k}(t)-\varphi_{d}(t)\right]=0 .
$$

The control (14) also admits a neural network interpretation skipped for brevity.

\section{Application}

In this section we consider a mechanical arm on a horizontal plane from [Futakata and Iwasaki, 2011], which is formed as a chain of three rigid links connected by two rotational joints to each other. The $i$-th link has the mass $m_{i}$ and the length $2 \ell_{i}(i=1,2,3)$, and the first link is connected to the inertial frame through a rotational joint. A spring of stiffness $k_{i}$ is mounted at the $i$-th joint, a dashpot of damping coefficient is $\rho k_{i}$, and each joint is connected to an actuator that generates the controlling torque $u_{i}$. As in [Futakata and Iwasaki, 2011] we selected:

$$
m_{i}=1, \ell_{i}=0.5, k_{i}=1, \rho=0.1 \text {. }
$$

First, let us take the linear part of the example from [Futakata and Iwasaki, 2011]:

$$
J=\frac{1}{12} I_{3}+Q, B=\left[\begin{array}{ccc}
1 & -1 & 0 \\
0 & 1 & -1 \\
0 & 0 & 1
\end{array}\right], C=B^{T}, S=B C
$$




$$
Q=L^{T} L, L=\left[\begin{array}{ccc}
0.5 & 0 & 0 \\
1 & 0.5 & 0 \\
1 & 1 & 0.5
\end{array}\right], \varrho=0.1,
$$

where $I_{3}$ is the identity matrix with dimension $3 \times 3$. This system has natural frequencies:

$$
\omega=\left[\begin{array}{lll}
5.146 & 1.915 & 0.292
\end{array}\right]^{T} .
$$

In Fig. 2 the results of the control (11) application is shown, where

$$
\mathcal{J}=\left\{\begin{array}{ll}
\{1,3\} \quad \text { if } t \leq 25 ; \\
\{2,3\} \quad \text { if } t>25,
\end{array} \quad U= \begin{cases}1 & \text { if } t \leq 25 \\
5 & \text { if } t>25\end{cases}\right.
$$

$\varepsilon=U / 3$ and $\eta=10$. Thus for $t \leq 25$ the system oscillates with the frequency $\omega_{2}=1.915$, next for $t>25$ the control (11) in a finite time activates the natural oscillations with $\omega_{1}=5.146$. Such a change of oscillation frequency (or a normal mode) corresponds to a transition from the "walking" mode to the "running" one in animals. As we can see from this figure, the canonical form variables $z$ are canceled or excited in a finite time corresponding to the chosen mode (one component of $z$ is oscillating the rest equal zero). For the state variable $x$ this results in the common frequency of oscillation change. Since the modes in $\mathcal{J}$ are canceled sufficiently fast (comparing with the system time scales), the frequencies in $\mathcal{J}$ almost do not influence on the $x$ behavior.
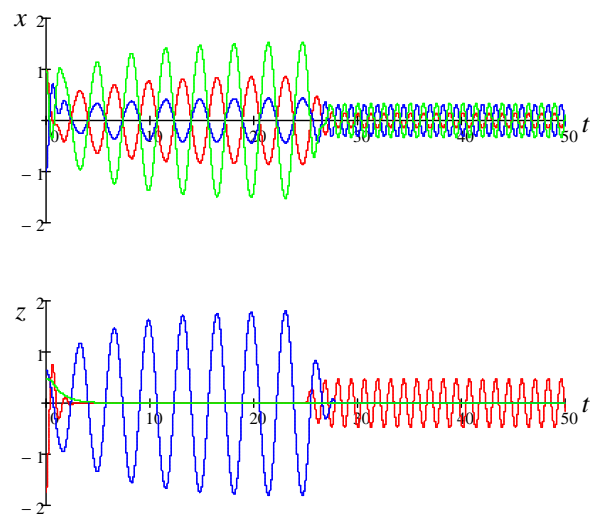

Fig. 2. The finite time control

The results of the control (14) application for the same time-varying set $\mathcal{J}$ and $U=1, E_{d}=1$, $\phi_{d}=\frac{2 \pi}{3}, \rho_{1}\left(\psi_{1}\right)=\left(U-2 \kappa_{k} \sqrt{E_{d}}\right) \tanh (5 \psi), \rho_{2}\left(\psi_{2}\right)=$ $\left(U-\kappa_{k} \sqrt{2 E_{d}}\right) \tanh (5 \psi)$ are shown in Fig. 3 (the trajectories $x(t)$ and $z(t)$ are given in Fig. 3,a and Fig. 3,b respectively, the energy variable $E$ is plotted in Fig. 3,c in the logarithmic scale; the phase variable $\varphi$ is presented in Fig. 3,d). As we can conclude, this control demonstrates a similar entrainment performance with additional phase resetting.

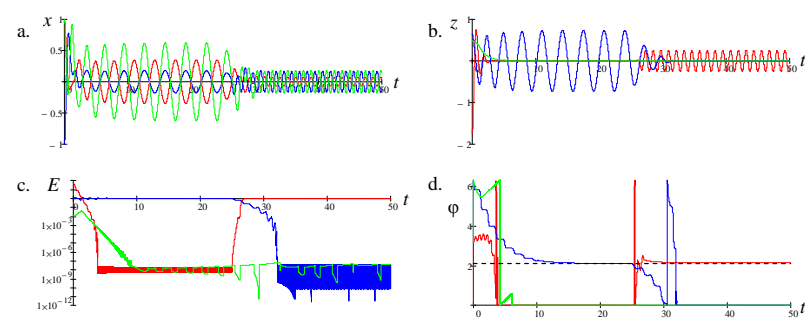

Fig. 3. The energy/phase regulation

The results of the controls (11) and (14) application confirm their entrainment abilities to the resonance oscillations at natural frequencies for (8). The switching between different frequencies (mode of oscillations) can be carried out by the incoming parameter setup $\left(U, \mathcal{J}, E_{d}\right.$ and $\phi_{d}$ ).

Now let us verify applicability of the proposed approach on nonlinear version of the example from [Futakata and Iwasaki, 2011]:

$$
J_{x}(x) \ddot{x}+G_{x}(x) \dot{x}^{2}+D \dot{x}+S x=B u,
$$

where $J_{x}(x)=\frac{1}{12} I_{3}+S_{x} Q S_{x}+C_{x} Q C_{x}$ and $G_{x}(x)=$ $S_{x} Q C_{x}-C_{x} Q S_{x}$ for $S_{x}=\operatorname{diag}\left\{\sin \left(x_{i}\right)\right\}, C_{x}=$ $\operatorname{diag}\left\{\cos \left(x_{i}\right)\right\}$, the rest matrices are the same as before. The results of the control (11) application with exactly the same parameters as in linear case are shown in Fig. 4. Comparing the curves presented in figures 2 and 4 it is possible note that the canonical form variables $z$ have the same qualitative behavior (one component is oscillating, others are canceled by the control). The cancellation is not exact for the system (15) due to appearance of nonlinear terms not covered by the control algorithm developed for the linear system (8). The oscillations are also less frequent due to additional dependence of frequencies of a nonlinear system on amplitude of oscillations. Computing numerically the average frequency of oscillations during the first 25 seconds we obtain 1.271 (1.915 in the linear case) and for $t \geq 25$ we get 3.607 (5.146 in the linear case). Nevertheless, the control (11) ensures the resonance mode activation and reliable switching among different frequencies even for sufficiently large deviations from the equilibrium point. However, the results of these simulations show that in a nonlinear case some quantitative deflections from the guaranteed in the linear case entrainment behavior can be expected.

\section{Conclusions}

The entrainment mechanism to a resonance mode based on CPG from [Futakata and Iwasaki, 2011], 

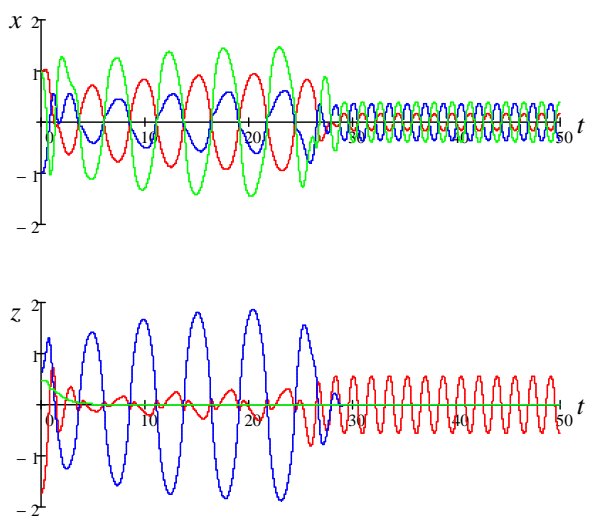

Fig. 4. The finite time control for nonlinear system

[Futakata and Iwasaki, 2008] is extended. Two control algorithms for feedback resonance, the finite-time control and the energy/phase regulation, are used to design entrainment neural networks. The obtained conditions of oscillation at a natural frequency are based on Lyapunov analysis and the optimal control theory (the harmonic balance method is avoided). One of the proposed controls provides the resonance oscillations in a finite time. The proposed approach can be applied to multi-DOF mechanical systems representable in the normal mode canonical form. The results of computer simulation demonstrate good entrainment ability of the proposed approach even for nonlinear systems.

Acknowledgments: The second author acknowledges the support of Russian Federal Program "Cadres" (Goscontracts 16.740.11.0042, 14.740.11.0942) and RFBR (project 11-08-01218). The third author gratefully acknowledges the support from the National Science Foundation under No.0654070 and No.1068997, and by the Office of Naval Research, under MURI Grant N00014-08-1-0642.

\section{References}

[Andrievsky, 2005] Andrievsky B.R. (2005). Computation of the excitability index for linear oscillators. Proc. ECC-CDC 2005, Seville, Spain, pp. 3537-3540.

[Delcomyn, 1980] Delcomyn F. (1980). Neural basis of rhythmic behavior in animals. Science, 210.

[Efimov and Fradkov, 2007a] Efimov D.V., Fradkov A.L. (2007). Hybrid adaptive resonance control using adaptive observers for vibration machines. Proc. ECC 2007, Kos, Greece, 26272634.

[Efimov and Fradkov, 2007b] Efimov D.V., Fradkov A.L. (2007). Hybrid adaptive resonance control using speed gradient approach for vibration machines. Proc. ACC 200\%, New-York, USA, pp. 3288-3293.

[Efimov and Fradkov, 2012] Efimov D.V., Fradkov A.L. Natural Wave Control in Lattices of Linear Oscillators. Systems \& Control Letters, 61, 2012, pp. 887-893.
[Efimov and Fridman, 2011] Efimov D.V., Fridman L. (2011). A Hybrid

Robust Non-Homogeneous Finite-Time Differentiator. IEEE Trans. Automatic Control, 56(5), 1213-1219.

[Filippov, 1988] Filippov A.F. (1988). Differential Equations with Discontinuous Right-hand Sides. Kluwer.

[Fradkov, 1999] Fradkov A.L. (1999). Exploring nonlinearity by feedback. Physica D, 128, 159-168.

[Andrievsky and Fradkov, 1999] Andrievsky B.R., Fradkov A.L. (1999). Feedback resonance in single and coupled 1-DOF oscillators. Int. J Bifurcations and Chaos, 10, pp. 2047-2058.

[Fradkov, 2007] Fradkov A.L. (2007). Cybernetical Physics: From Control of Chaos to Quantum Control. Springer.

[Futakata and Iwasaki, 2011] Futakata Y., Iwasaki T. (2011). Entrainment to natural oscillations via uncoupled central pattern generators. IEEE Trans. Automatic Control, 56(5), 1075-1089.

[Futakata and Iwasaki, 2008] Futakata Y., Iwasaki T. (2008). Formal analysis of resonance entrainment by central pattern generator. Journal of Mathematical Biology, 57(2), 183-207.

[Desch et al., 2001] Desch W., Logemann H., Ryan E.P., Sontag E.D. (2001). Meagre functions and asymptotic behaviour of dynamical systems. Nonlinear Anal., 44(8, Ser. A: Theory Methods), 1087-1109.

[Geering, 2007] Geering H.P. (2007). Optimal Control with Engineering Applications. Springer.

[Grillner et al., 1988] Grillner S., J.T. Buchanan, P. Walker, Brodin L. (1988). Neuronal Control of Rhythmic Movements in Vertebrates. New York: Wiley.

[Holt et al., 1991] Holt K.G., Hamill J., Anders R.O. (1991). Predicting the minimal energy costs of human walking. Medicine and Science in Sport and Exercise, 23(4), 491-498.

[Orlovsky et al., 1999] Orlovsky G.N., T.G. Deliagina, Grillner S. (1999). Neuronal Control of Locomotion: From Mollusc to Man. Oxford University Press.

[Shiriaev, 2000] Shiriaev A.S. (2000). The notion of Vdetectability and stabilization of invariant sets of nonlinear systems. Systems \& Control Letters, 39(5), 327-338.

[Shiriaev and Fradkov, 2001] Shiriaev A.S., Fradkov A.L. (2001). Stabilization of invariant sets for nonlinear systems with applications to control of oscillations. Int. J. Robust Nonlinear Control, 11, pp. 215-240.

[Yakubovich et al., 2004] Yakubovich V.A., Leonov G.A., Gelig A.Kh. (2004). Stability of Stationary Sets in Control Systems with Discontinuous Nonlinearities. Singapure: World Scientific. 